\title{
ON THE GENERATION OF FAIR-WEATHER ELECTRIC FIELD IN ATMOSPHERES OF ROTATING PLANETS WITH COMPLEX INTERIOR
}

\author{
P. A. Bespalov, Yu. V. Chugunov and S. S. Davydenko \\ Department of Astrophysics and Space Plasma Physics \\ Institute of Applied Physics, Russian Academy of Science \\ 46 Ulyanov st., 603600 Nizhny Novgorod, Russia
}

\begin{abstract}
An additional mechanism of generation of the atmospheric electricity and formation of the global current circuit in magnetospheres of rotating planets is considered. An advanced model of the planetary electric generator taking into account complex inner structure of the planet is developed. For simplicity's sake, the case of homogeneously conducting atmosphere under the global fair-weather is investigated. It is assumed that there is a uniformly magnetized core inside the planet rotating at angular velocity generally not equal to the planetary angular velocity. In the considered axisymmetric model of the inner planetary flows, the effect of the planetary electric generator is stipulated by potential part of sources of the magnetic field. As shown, a difference of the core's and planetary angular velocities leads to change of a global voltage applied to the atmospheric layer in comparison with the case of co-rotation between the core and planet. A contribution of current system of the planetary electric generator to the total magnetic field is estimated. As qualitatively applied to the terrestrial conditions, the considered model of the planetary electric generator gives a ground-ionosphere voltage no less than one third of the observed value and must be considered equally with such well-known sources of the atmospheric electricity as thunderstorm activity and magnetospheric macro processes. An application of the developed approach to the case of Jovian magnetosphere is also discussed.
\end{abstract}

\section{Introduction}

A problem of sources of the atmospheric electricity is still a subject of investigations and active discussions. The most well known hypothesis describing the electric global phenomena was suggested by Wilson in the beginning of the century. Since the Wilson's work (1925), thunderstorms are considered usually as a main source of the atmospheric electricity. In accordance with the Wilson's theory, thunderstorms play a role of the electric generators in the global current circuit. In the average, separation of electric charges occurs in thunderstorm cloud: negative charges are carried toward the ground, whereas positive charge is transferred to the well-conducting layers in the upper atmosphere. The positive and negative charges distribute homogeneously over the Earth's surface and upper atmosphere respectively. Owing to the above charge separation, a capacitor-like electric field driving a downward current in all fair-weather regions is built up in the atmosphere. However, this model could not explain some experimental results on the atmospheric electricity, for example, an existence of the horizontal electric field in the atmosphere. To reach an agreement between the theoretical model and experimental measurements, a number of additional mechanisms of generation of the atmospheric electricity were suggested. As sources of the electric field in the atmosphere, these models considered both macro processes in the ionosphere and magnetosphere (Volland, 1977) and a different mechanisms of electric charges separation in the atmosphere outside thunderstorm clouds (Reiter, 1985).

The aim of this article is to consider an additional mechanism of generation of the 
atmospheric electricity based on the model of the planetary electric generator suggested by Bespalov and Chugunov (1996). As shown earlier (Bespalov et al., 1996), this mechanism ensures a voltage between the ground and ionosphere about the unipolar potential, which is approximately equal to $95 \mathrm{kV}$ under the terrestrial conditions. Taking into consideration an altitude dependence of the atmospheric conductivity, this model also gives the fair-weather electric current density at the surface of the planet about $10^{-12} \mathrm{~A} / \mathrm{m}^{2}$, which is close to the observed value, and reasonable altitude distributions of the electric field and current density in the atmospheric layer. However, the above consideration was made under some simplifying assumptions. In particular, it was assumed that the planet has homogeneous interior and rotates as a solid body. As known, it is not the case for the Earth, where there is a hot liquid core in the center of the planet (Love and Bloxham, 1994). In this article, we investigated an influence of the complex inner structure of the planet on generation of the atmospheric electricity in the frame of Earth-like model of the planetary interior. Namely, we assumed that there is a magnetized conducting core inside the planet.

The paper is organized as follows. In the second part of the article, we considered a model of the planetary electric generator taking into account an existence of the uniformly magnetized core inside the planet. A set of general equations describing the electrodynamics of the planetary generator is written, and boundary conditions in the problem as well as a correct choice of the frame of reference are discussed. In the third part, we obtained an exact solution of the above problem under the model case of homogeneous conductivity of the atmosphere. Qualitative estimates are made to show an influence of the inner planetary core on the induced voltage between the ground and upper atmosphere. In the last section, the results obtained were qualitatively applied to the terrestrial conditions. We also discussed the further development of the model and, finally, summarized the main conclusions.

2. A Model of the Planetary Electric Generator and Basic Equations

To consider the most important features of the global electrodynamics of the planetary electric generator, let us investigate the following model problem. Assume that a planet of radius $r_{0}$ rotates at the angular velocity $\omega_{0}$ and has a uniform electric conductivity $\sigma_{0}$. There is a homogeneously magnetized core in the center of the planet. A radius of the core is $r_{c}$, electric conductivity is $\sigma_{c}$ and angular velocity is $\omega_{c}$. It should be pointed out that rotational axis of the core coincides with the planetary rotational axis, but their angular velocities can differ. Magnetic moment of the core $\mathbf{M}$ is also directed along the rotational axis. Planetary atmosphere has a total height $h$ and rigidly co-rotates with the planet at the angular velocity $\omega_{0}$. Electric conductivity of the atmosphere $\sigma_{a}$ is assumed to be constant and does not depend on both altitude over the planetary surface and latitude. As the upper boundary of the atmosphere, a sphere of radius $r_{a}=r_{0}+h$ serves. Outbound the atmosphere, planetary plasma envelope is placed. It is assumed that the plasma envelope rotates as a solid body at the angular velocity $\boldsymbol{\omega}_{m}$ and has a homogeneous isotropic electric conductivity $\sigma_{m}$. The above model of the planetary electric generator is plotted in Fig.1.

To determine distributions of the electric potential $\phi$, electric charge density $\rho$ and current density $\mathbf{j}$ ensured by the planetary electric generator, we should solve the following set of the electrodynamic equations:

$$
\begin{aligned}
& \mathbf{j}=\sigma\left(-\nabla \phi+\frac{\mathbf{v} \times \mathbf{B}}{c}\right)+\rho \mathbf{v} \\
& \nabla \cdot \mathbf{j}=0 \\
& \Delta \phi=-4 \pi \rho,
\end{aligned}
$$

where $\mathbf{B}$ is a magnetic field, $c$ is the speed of light and $\mathbf{v}$ is an azimuthal velocity of the medium determined by the local angular velocity. 


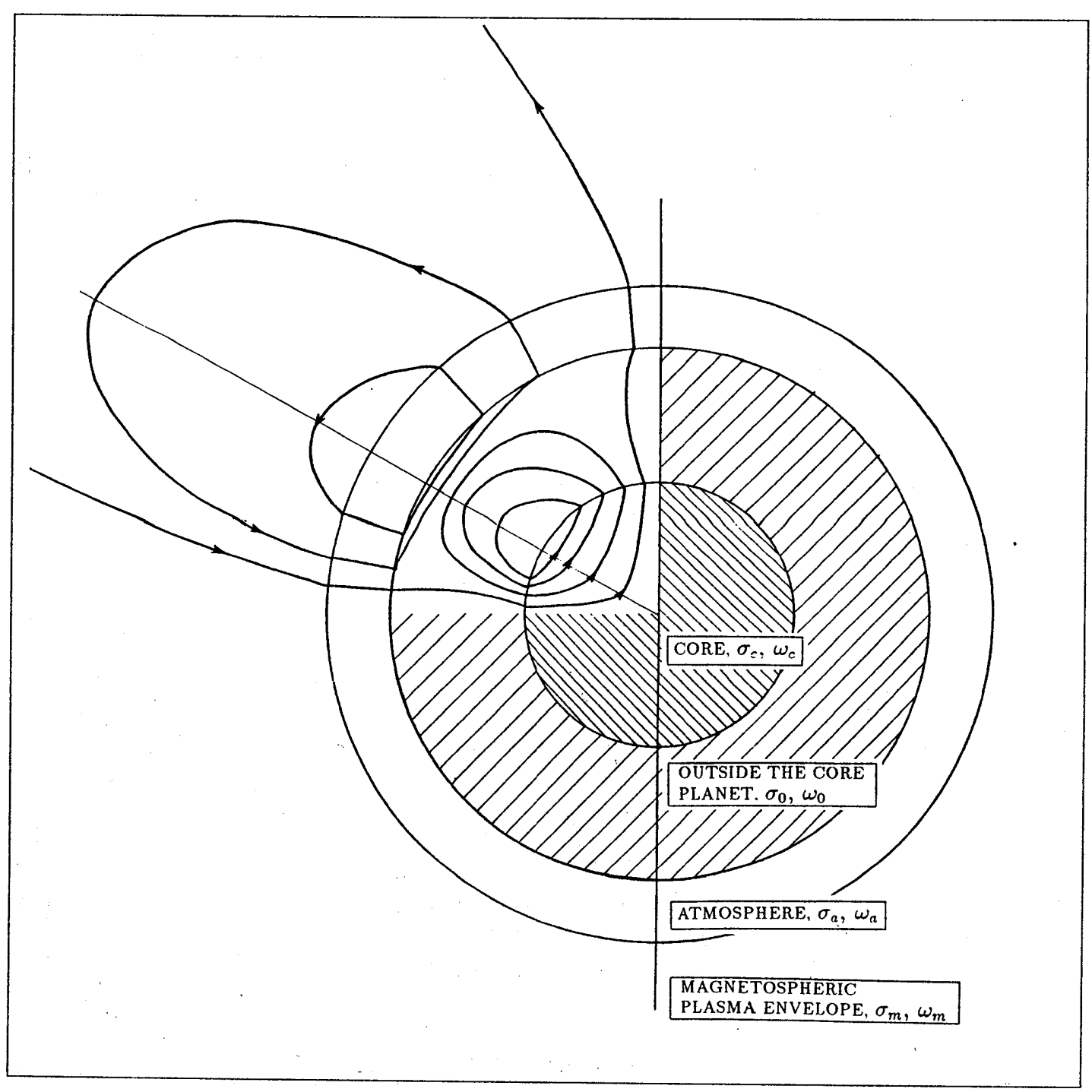

Fig.1. Qualitative plot of the planetary electric generator with uniform atmospheric conductivity and homogeneously magnetized core. Current system of the planetary generator is shown in selected quarter for the case $\delta_{c}>0, M \uparrow \downarrow \omega_{0}$.

In the suggested model, magnetic field $\mathbf{B}$ is assumed to be determined by magnetization of the core and by a current system of the planetary generator. Of course, the real situation can be quite different as compared to this model. For example, it is generally believed that the terrestrial planetary magnetic field is produced by the magnetic dynamo in the liquid core and this mechanism of the magnetic field generation is sufficient only in the case of finite angle between the magnetic and rotational axes (Parker, 1979). It is also known that terresstrial core rotates differentially, so angular velocity of the media increases when approaching to the rotational axis (Lefftz et al., 1994). However, the above mentioned features of the magnetic field structure and media flows would be important for the analysis of the planetary magnetic field generation that is not a subject 
of the article. Since we are not interested in the nature of the planetary magnetic field, the real magnetic field source can be replaced, as a model, by uniformly magnetized core rotating rigidly on the magnetic axis at the averaged angular velocity.

It is easily seen that even in the frame of this simple model, it is rather difficult to solve the problem exactly with the self-consistent magnetic field. To simplify the calculations, let us use the method of successive approximations: in the beginning, we suppose that magnetic field is determined by magnetization of the planetary core only. In the case of homogeneous magnetization of the core, magnetic field is uniform inside the core and equal to $2 \mathrm{M} / r_{c}^{3}$. Outside the core, magnetic field coincides with the field of magnetic dipole $\mathbf{M}$ placed in the center of the planet. Using the solution of the problem with the given magnetic field, we can estimate a contribution of the current system to the total magnetic field.

It should be also pointed out that we can use the well-known set of electrodynamic equations (1) in the inertial frame only. If the frame of reference rotates at the angular velocity $\Omega$, the ordinary electrodynamic equations (1) generally have another form (Panofsky and Phillips, 1969). However, if a solution of the problem is obtained in the inertial frame, it is possible to determine the electric field $\mathbf{E}^{\prime}$ and current density $\mathbf{j}^{\prime}$ in the slowly rotating frame by means of the local Lorentz transformation (Landau et al., 1984)

$$
\begin{gathered}
\mathbf{E}^{\prime}=\mathbf{E}+\frac{(\boldsymbol{\Omega} \times \mathbf{r}) \times \mathbf{B}}{c} \\
\mathbf{j}^{\prime}=\mathbf{j}-\rho(\boldsymbol{\Omega} \times \mathbf{r}) .
\end{gathered}
$$

Now, let us find the solution in the inertial frame. Under the consideration, it is convenient to use a spherical frame $(r, \theta, \varphi)$ with the reference point in the center of the planet. As follows from the set of equations (1), the electric potential satisfies the following nonuniform equation

$$
\Delta \phi=\frac{1}{c} \nabla \cdot(\mathbf{v} \times \mathbf{B})
$$

and substantially depends on distribution of the magnetic field. As assumed above, magnetic field is produced by homogeneous magnetization of the inner planetary core.

Let us consider a general solution of the equation (3) inside the core. Bearing in mind that magnetic field in the core is homogeneous, the equation (3) takes the form

$$
\Delta \phi=\frac{4 M \omega_{c}}{r_{c}^{3} c} .
$$

We shall look for a solution of the equation (4) in the following form:

$$
\phi=\phi_{0}+\frac{M \omega_{c}}{r_{c}^{3} c} r^{2} \sin ^{2} \theta+\mathcal{F}(r)\left(3 \cos ^{2} \theta-1\right),
$$

where $\theta$ is a co-latitude angle, $\phi_{0}$ is a constant, $\mathcal{F}(r)$ is unknown function. Substituting the expression $(5)$ in the general equation $(3)$, one can obtain that the function $\mathcal{F}(r)$ satisfies the well-known Lagrange's equation

$$
r^{2} \frac{d^{2} \mathcal{F}(r)}{d r^{2}}+2 r \frac{d \mathcal{F}(r)}{d r}-6 \mathcal{F}(r)=0
$$

A general solution of the second-order ordinary differential equation (6) can be written as

$$
\mathcal{F}(r)=A_{1} r^{-3}+A_{2} r^{2}
$$


where constants $A_{1}$ and $A_{2}$ are determined by the boundary conditions.

As shown before, magnetic field is assumed to be dipole in the planet ouside the core, in the atmosphere and in the plasma envelope. In these regions, the general equation (3) can be reduced to

$$
\Delta \phi=\frac{M \omega}{c r^{3}}\left(3 \cos ^{2} \theta-1\right),
$$

where $\omega$ is the local angular velocity of the medium. It is convenient to use the following form of solution of the equation (8):

$$
\phi=\phi_{0}+\frac{M \omega}{c} \frac{\sin ^{2} \theta}{r}+\mathcal{Q}(r)\left(3 \cos ^{2} \theta-1\right),
$$

where $\phi_{0}$ is a constant, $\mathcal{Q}(r)$ is unknown function. Substituting chosen form of the solution (9) in the equation (8), one can see that, as before, unknown function $\mathcal{Q}(r)$ satisfies the Lagrange's equation (6) and is described by the expression (7), where the constants $A_{1}$ and $A_{2}$ are determined by the boundary conditions of the equation (8).

To obtain a distribution of the electric potential $\phi$, it is necessary to define a boundary conditions of the equation (3). It seems natural to assume that the potential must be continuous anywhere and ensure a continuity of radial component of the electric current density at boundaries of the media with different electric conductivity. We also assume that the potential has no singularities and tends to zero at the infinity.

Taking into account the described above boundary conditions and general forms of the solution both inside (5) and outside the core (9), one can obtain a distribution of the electric potential in whole of the space. An exact Distribution of the electric potential $\phi$ is as follows in the core

$$
\begin{aligned}
\frac{\phi}{U_{0}}= & -\frac{2}{3}\left(\delta_{m} x_{a}^{-1}+\delta_{c} \alpha^{-1}\right)+\left(\delta_{c}+1\right) \alpha^{-3} \sin ^{2} \theta x^{2}+x^{2} \frac{3 \cos ^{2} \theta-1}{2} \times \\
& \frac{\frac{5}{2} \alpha^{-5}}{\left(1-\frac{\sigma_{c}}{\sigma_{0}}\right)\left(1+\eta_{c} \alpha^{-5}\right)}\left\{\frac{2}{3} \delta_{c} \alpha^{-3}\left(\frac{3}{5}+\frac{2}{5} \alpha^{5}\right)+\frac{\frac{2}{3} \delta_{m} x_{a}^{2}}{1-\frac{\sigma_{a}}{\sigma_{m}}}+\mathcal{A}\left(1-x_{a}^{5} \eta_{a}\right)\right\}
\end{aligned}
$$

in the planet outside the core

$$
\begin{aligned}
& \frac{\phi}{U_{0}}=-\frac{2}{3} \delta_{m} x_{a}^{-1}+\frac{\sin ^{2} \theta}{x}+\frac{3 \cos ^{2} \theta-1}{2} \frac{1}{1+\eta_{c} \alpha^{-5}} \times \\
& \left\{x^{2} \alpha^{-5} \eta_{c}\left(\frac{\frac{2}{3} \delta_{m} x_{a}^{2}}{1-\frac{\sigma_{a}}{\sigma_{m}}}+\mathcal{A}\left(1-x_{a}^{5} \eta_{a}\right)+\frac{\frac{2}{3} \delta_{c} \alpha^{2}}{1+\frac{3}{2} \frac{\sigma_{0}}{\sigma_{c}}}\right)+\right. \\
& \left.x^{-3}\left(\frac{\frac{2}{3} \delta_{m} x_{a}^{2}}{1-\frac{\sigma_{a}}{\sigma_{m}}}+\mathcal{A}\left(1-r_{a}^{5} \eta_{a}\right)+\frac{\frac{2}{3} \delta_{c} \alpha^{-3}}{1-\frac{\sigma_{0}}{\sigma_{c}}}\right)\right\},
\end{aligned}
$$

in the atmosphere

$$
\frac{\phi}{U_{0}}=-\frac{2}{3} \delta_{m} x_{a}^{-1}+\frac{\sin ^{2} \theta}{x}+\frac{3 \cos ^{2} \theta-1}{2}\left\{\mathcal{A} x^{2}+x^{-3}\left(\frac{\frac{2}{3} \delta_{m} x_{a}^{2}}{1-\frac{\sigma_{a}}{\sigma_{m}}}-\mathcal{A} x_{a}^{5} \eta_{a}\right)\right\}
$$

and in the plasma envelope

$$
\frac{\phi}{U_{0}}=\left(1-\delta_{m}\right) \frac{\sin ^{2} \theta}{x}+x^{-3} \frac{3 \cos ^{2} \theta-1}{2}\left\{\frac{\frac{2}{3} \delta_{m} x_{a}^{2}}{1-\frac{\sigma_{a}}{\sigma_{m}}}+\mathcal{A} x_{a}^{5}\left(1-\eta_{a}\right)\right\} .
$$


Here

$$
\begin{array}{r}
x_{a}=\frac{r_{a}}{r_{0}}, \quad \alpha=\frac{r_{c}}{r_{0}}, \quad \delta_{c}=\frac{\omega_{c}-\omega_{0}}{\omega_{0}}, \quad \delta_{m}=\frac{\omega_{0}-\omega_{m}}{\omega_{0}}, \quad U_{0}=\frac{M \omega_{0}}{r_{0} c}, \\
\eta_{c}=\frac{1+\frac{3}{2} \frac{\sigma_{0}}{\sigma_{c}}}{\frac{\sigma_{0}}{\sigma_{c}}-1}, \quad \eta_{0}=\frac{3-2 \eta_{c} \alpha^{-5}+2 \frac{\sigma_{a}}{\sigma_{0}}\left(1+\eta_{c} \alpha^{-5}\right)}{3-2 \eta_{c} \alpha^{-5}-3 \frac{\sigma_{a}}{\sigma_{0}}\left(1+\eta_{c} \alpha^{-5}\right)}, \quad \eta_{a}=\frac{1+\frac{2}{3} \frac{\sigma_{a}}{\sigma_{m}}}{1-\frac{\sigma_{a}}{\sigma_{m}}} \\
\mathcal{A}=\frac{1}{x_{a}^{5} \eta_{a}-\eta_{0}}\left\{\frac{3-2 \eta_{c} \alpha^{-5}}{1+\frac{3}{2} \alpha^{5}+\frac{3}{2} \frac{\sigma_{0}}{\sigma_{c}}\left(1-\alpha^{5}\right)} \frac{\frac{2}{3} \delta_{m} x_{a}^{2}}{3-2 \eta_{c} \alpha^{-5}-3 \frac{\sigma_{a}}{\sigma_{0}}\left(1+\eta_{c} \alpha^{-5}\right)}+\frac{\sigma_{a}}{\sigma_{m}}\right\} .
\end{array}
$$

Using the drawn above distribution of the electric potential, one can also obtain a distribution of radial and co-latitude components of the electric current density $j_{r}$ and $j_{\theta}$ in the core

$$
\begin{gathered}
j_{r}=-\sigma_{c} \frac{U_{0}}{r_{0}} x \frac{3 \cos ^{2} \theta-1}{2} \frac{5 \alpha^{-5}}{\left(1-\frac{\sigma_{c}}{\sigma_{0}}\right)\left(1+\eta_{c} \alpha^{-5}\right)} \times \\
\left\{\frac{2}{3} \delta_{c} \alpha^{-3}\left(\frac{3}{5}+\frac{2}{5} \alpha^{5}\right)+\frac{\frac{2}{3} \delta_{m} x_{a}^{2}}{1-\frac{\sigma_{a}}{\sigma_{m}}}+\mathcal{A}\left(1-x_{a}^{5} \eta_{a}\right)\right\} \\
j_{\theta}=\sigma_{c} \frac{U_{0}}{r_{0}} x \cos \theta \sin \theta \frac{\frac{5}{2} \alpha^{-5}}{\left(1-\frac{\sigma_{c}}{\sigma_{0}}\right)\left(1+\eta_{c} \alpha^{-5}\right)}\left\{2 \delta_{c} \alpha^{-3}\left(\frac{3}{5}+\frac{2}{5} \alpha^{5}\right)+\right. \\
\left.\frac{2 \delta_{m} x_{a}^{2}}{1-\frac{\sigma_{a}}{\sigma_{m}}}+3 \mathcal{A}\left(1-x_{a}^{5} \eta_{a}\right)\right\}
\end{gathered}
$$

in the planet outside the core

$$
\begin{array}{r}
j_{r}=-\sigma_{0} \frac{U_{0}}{r_{0}} \frac{3 \cos ^{2} \theta-1}{2} \frac{1}{1+\eta_{c} \alpha^{-5}}\left\{2 x \alpha ^ { - 5 } \eta _ { c } \left(\frac{\frac{2}{3} \delta_{m} x_{a}^{2}}{1-\frac{\sigma_{a}}{\sigma_{m}}}+\mathcal{A}\left(1-x_{a}^{5} \eta_{a}\right)+\right.\right. \\
\left.\left.\frac{\frac{2}{3} \delta_{c} \alpha^{2}}{1+\frac{3}{2} \frac{\sigma_{0}}{\sigma_{c}}}\right)-3 x^{-4}\left(\frac{\frac{2}{3} \delta_{m} x_{a}^{2}}{1-\frac{\sigma_{a}}{\sigma_{m}}}+\mathcal{A}\left(1-r_{a}^{5} \eta_{a}\right)+\frac{\frac{2}{3} \delta_{c} \alpha^{-3}}{1-\frac{\sigma_{0}}{\sigma_{c}}}\right)\right\} \\
j_{\theta}=\sigma_{0} \frac{U_{0}}{r_{0}} \cos \theta \sin \theta \frac{1}{1+\eta_{c} \alpha^{-5}}\left\{x \alpha^{-5} \eta_{c}\left(\frac{2 \delta_{m} x_{a}^{2}}{1-\frac{\sigma_{a}}{\sigma_{m}}}+3 \mathcal{A}\left(1-x_{a}^{5} \eta_{a}\right)+\frac{2 \delta_{c} \alpha^{2}}{1+\frac{3}{2} \frac{\sigma_{0}}{\sigma_{c}}}\right)+\right. \\
\left.x^{-4}\left(\frac{2 \delta_{m} x_{a}^{2}}{1-\frac{\sigma_{a}}{\sigma_{m}}}+3 \mathcal{A}\left(1-r_{a}^{5} \eta_{a}\right)+\frac{2 \delta_{c} \alpha^{-3}}{1-\frac{\sigma_{0}}{\sigma_{c}}}\right)\right\}
\end{array}
$$

in the atmosphere

$$
\begin{gathered}
j_{r}=-\sigma_{a} \frac{U_{0}}{r_{0}} \frac{3 \cos ^{2} \theta-1}{2}\left\{2 x \mathcal{A}-3 x^{-4}\left(\frac{\frac{2}{3} \delta_{m} x_{a}^{2}}{1-\frac{\sigma_{a}}{\sigma_{m}}}-\mathcal{A} x_{a}^{5} \eta_{a}\right)\right\} \\
j_{\theta}=\sigma_{a} \frac{U_{0}}{r_{0}} \cos \theta \sin \theta\left\{3 x \mathcal{A}+x^{-4}\left(\frac{2 \delta_{m} x_{a}^{2}}{1-\frac{\sigma_{a}}{\sigma_{m}}}-3 \mathcal{A} x_{a}^{5} \eta_{a}\right)\right\}
\end{gathered}
$$


and in the plasma envelope

$$
\begin{gathered}
j_{r}=\sigma_{m} \frac{U_{0}}{r_{0}} x^{-4} \frac{3 \cos ^{2} \theta-1}{2}\left\{\frac{2 \delta_{m} x_{a}^{2}}{1-\frac{\sigma_{a}}{\sigma_{m}}}+3 \mathcal{A} x_{a}^{5}\left(1-\eta_{a}\right)\right\} \\
j_{\theta}=\sigma_{m} \frac{U_{0}}{r_{0}} \cos \theta \sin \theta x^{-4}\left\{\frac{2 \delta_{m} x_{a}^{2}}{1-\frac{\sigma_{a}}{\sigma_{m}}}+3 \mathcal{A} x_{a}^{5}\left(1-\eta_{a}\right)\right\} .
\end{gathered}
$$

A sketch of current system of the planetary electric generator is shown in Fig.1.

It seems interesting to apply the obtained results to the terrestrial conditions. However, the exact solution was obtained in the case of homogeneous conductivity of the atmosphere, whereas the terrestrial atmosphere has substantially nonuniform electric conductivity (Volland, 1987). Therefore, the above expressions can be applied to the Earth's conditions qualitatively and describe the main features of the global atmospheric electricity only.

As seen from the formulas (10)-(17), an exact solution of the problem is relatively complicated. However, in application to the terrestrial conditions, it becomes possible to simplify the obtained expressions considerably. First, let us take into account that a total height of the atmosphere $h$ is about $10^{7} \mathrm{~cm}$ and substantially less than the planetary radius $r_{0}$, which is approximately equal to $6.37 \cdot 10^{8} \mathrm{~cm}$. Second, conductivity of the atmosphere $\sigma_{a}$ is less than conductivities of the core $\sigma_{c}$, mantle $\sigma_{0}$ and plasma envelope $\sigma_{m}$ (Volland, 1987). We also assume that conductivity of the core is much greater than mantle's conductivity (Love and Bloxham, 1994). In other words, the following inequalities are valid under the terrestrial conditions

$$
\frac{\sigma_{0}}{\sigma_{c}} \ll\left\{\frac{\sigma_{a}}{\sigma_{0}}, \frac{\sigma_{a}}{\sigma_{m}}\right\} \ll \frac{h}{r_{0}} \ll 1
$$

where conductivity of the atmosphere $\sigma_{a}$, as a model, is assumed to be equal to the atmospheric conductivity at the surface of the Earth.

Taking into account the inequalities (18), the exact solutions (10)-(17) can be simplified considerably. First of all, it is of great interest to obtain rough expressions describing the main parameters of the atmospheric electricity. An exact distribution of the electric potential in the atmosphere (12) can be reduced to the following relatively simple formula:

$$
\frac{\phi}{U_{0}} \approx \sin ^{2} \theta\left(1-\delta_{m} \frac{z}{h}+\frac{5 \delta_{c} \alpha^{2}}{2+3 \alpha^{5}}\left(1-\frac{z}{h}\right)\right)-\frac{2}{3}\left(\delta_{m}+\frac{5 \delta_{c} \alpha^{2}}{2+3 \alpha^{5}}\right)\left(1-\frac{z}{h}\right)
$$

where $z=r-r_{0}$ is an altitude over the planetary surface. As seen from the expression (19), the ground-ionosphere voltage $\phi\left(r_{0}+h\right)-\phi\left(r_{0}\right)$ does not depend on the thickness of the atmosphere and is about

$$
U_{\text {eff }}=U_{0}\left(1+\frac{5 \delta_{c} \alpha^{2}}{2+3 \alpha^{5}}\right)
$$

where $U_{0}=\omega_{0} M / r_{0} c$ is the unipolar potential arising owing to planetary rotation. So long as magnetic moment of the Earth $M$ is about $8.3 \cdot 10^{25} \mathrm{G} \cdot \mathrm{cm}^{3}$ and angular velocity of the planet $\omega_{0}$ is $7.3 \cdot 10^{-5} \mathrm{rad} \mathrm{s}^{-1}$, a value of the terrestrial unipolar potential is close to $95 \mathrm{kV}$. As seen, the voltage (20) between the ground and ionosphere can be not equal to the ground-ionosphere voltage $U_{0}$ calculated in the case of rigidly rotating planet with uniform inner structure (Bespalov and Chugunov, 1996). A difference of angular velocities 
between the core and mantle modify a global voltage applied to the atmosphere. Under the terrestrial conditions, differential rotation of the core with respect to the mantle is of the order of $10^{-10} \mathrm{rad} \mathrm{s}^{-1}$ (Lefftz et al., 1994). In that case $\delta_{c} \ll 1$ and, as follows from the expression $(20)$, the terrestrial ground-ionosphere voltage $U_{\text {eff }}$ only slightly differs from the $U_{0} \approx 95 \mathrm{kV}$. However, it is not the case for the Jupiter, where magnetized core rotates faster than outer shells of the planet and the considered before effect can be substantial (Stevenson and Salpeter, 1976).

In the case of homogeneous atmospheric conductivity, electric field in the atmosphere is mainly radial, approximately uniform and about $U_{\text {eff }} / h$. As follows from the local Lorentz transformation (2), radial electric field in the co-rotating frame of reference $E_{r}^{\prime}$ is equal to $(\mathbf{E}+\mathbf{v} \times \mathbf{B} / c)_{r}$. One can easily check that an additional electric field $\mathbf{v} \times \mathbf{B} / c$ arising owing to the transformation is of the order of $U_{0} / r_{0}$ and much less than radial electric field in the inertial frame $E_{r}=U_{\text {eff }} / h$. Therefore, atmospheric electric field in the co-rotating frame $E_{r}^{\prime} \approx U_{0} / r_{0}+U_{\text {eff }} / h \approx E_{r}$.

Using the inequalities (18), one can also obtain a simplified formula describing a distribution of radial component of the electric current density in the atmosphere

$$
j_{r} \approx \sigma_{a}\left(\sin ^{2} \theta-\frac{2}{3}\right) \frac{U_{0}}{h}\left(\delta_{m}+\frac{5 \delta_{c} \alpha^{2}}{1+3 \alpha^{5}}\right) .
$$

As seen, current density in the uniformly conducting atmosphere is determined by the atmospheric conductivity and applied global voltage, and is about $\sigma_{a} U_{\text {eff }} / h$.

\section{Discussion and Conclusions}

In the beginning of this section, we would like to consider in more detail some points of the described above model. First of all, let us remind that magnetic field in the problem was assumed to be homogeneous inside the core and dipole outside it. This distribution of the magnetic field coincides with the magnetic field of non-rotating homogeneously magnetized core. However, planetary electric generator has own current system, which can disturb the core's magnetic field. First, convective current $\rho \mathbf{v}$ arising owing to the planetary rotation can make a contribution to the total magnetic moment. Second, quadrupole currents are built up inside the planet, where the magnetized core rotates with respect to the well-conducting mantle. These quadrupole currents produce an azimuthal magnetic field, which also was not taken into account in the model. Now, we consider both of these sources of the additional magnetic field.

First, a contribution of the convective current $\rho \mathbf{v}$ to the initial magnetic field have to be estimated. As was assumed before, azimuthal velocities of the media $v$ are determined by local angular velocities, electric charge density in the core $\rho_{c}$ and in the mantle $\rho_{0}$ can be obtained from the Poisson equation $\Delta \phi=-4 \pi \rho$ and equations (4) and (8):

$$
\rho_{c}=-\frac{M \omega_{c}}{\pi c r_{c}^{3}}, \quad \rho_{0}=-\frac{M \omega_{0}}{4 \pi c r^{3}}\left(3 \cos ^{2} \theta-1\right) .
$$

It is clear that this azimuthal current creates its own dipole magnetic moment in addition to magnetic moment of the core. Using expression (22) and a well-known formula determining a total magnetic moment $\Delta \mathbf{M}$ of a stable current system $\mathbf{j}$ flowing inside a finite volume $V$

$$
\Delta \mathbf{M}=\frac{1}{2 c} \int \mathbf{r} \times \mathbf{j} d V
$$

we can obtain a ratio of the magnetic moment $\Delta M_{\text {conv }}$ produced by the convective current to the magnetic moment of the core:

$$
\frac{\Delta M_{\text {conv }}}{M}=-\frac{\omega_{0}^{2} r_{0}^{2}}{15 c^{2}}\left(1-\alpha^{2}\right)-\frac{4 \omega_{c}^{2} r_{c}^{2}}{15 c^{2}} \sim\left(\frac{v_{\varphi}}{c}\right)^{2} \ll 1 .
$$


As seen from the relation (23), a contribution of the convective current to the core's magnetic field can be neglected under the terrestrial conditions.

As was mentioned above, the quadrupole currents can also modify magnetic field produced by magnetization of the core. It should be noticed that in the case of co-rotation between the core and mantle, all of the quadrupole currents flow through the atmosphere. Under the terrestrial conditions, electric conductivity of the atmosphere is relatively low and the currents are rather slight to make a considerable addition to the core's magnetic field (Bespalov et al., 1996). Therefore, we must investigate in more detail an influence of the quadrupole currents appearing inside the well-conducting planet owing to difference of angular velocities of the planet and core. To determine a total magnetic field of the planetary electric generator taking into account the field connected with the quadrupole currents, let us simplify the problem and determine magnetic field induced by homogeneously magnetized sphere of radius $r_{c}$ rotating at angular velocity $\omega_{c}-\omega_{0}$ in immovable conducting medium. As before, electric conductivity of the sphere $\sigma_{c}$ is assumed to be much greater than conductivity of the surrounding medium $\sigma_{0}$. In the frame of this simplified model, one can derive that the magnetic field can be relatively easy presented as a sum of potential and vortex components. Since an axisymmetric model of the planetary interior is assumed, generation of quadrupole currents and, respectively, the effect of the planetary electric generator are caused by interaction of flows of the conducting media with the potential component of the magnetic field. In this connection, chosen before model of the planetary interior, where there is a uniformly magnetized sphere inside the planet, seems quite acceptable. In that case, the potential magnetic field coincides with the field produced by magnetization of the core, whereas the vortex magnetic field is determined by the quadrupole currents only and does not exert a substantial influence on performance of the planetary generator.

In accordance with the above consideration, we can neglect a contribution of the convective current to the potential magnetic field and obtain a total current $I$ flowing in the quadrupole loop:

$$
I=\frac{4 \pi}{3 \sqrt{3}} \frac{\sigma_{0}}{1+\frac{3 \sigma_{0}}{2 \sigma_{c}}} \frac{M \delta_{c} \omega_{0}}{c} \approx \frac{4 \pi \sigma_{0}}{3 \sqrt{3}} \frac{M \delta_{c} \omega_{0}}{c} .
$$

Using the expression (24), one can estimate a maximal azimuthal magnetic field produced by the quadrupole currents:

$$
B_{\varphi}^{\max }=\frac{\sqrt{6} I}{c r_{c}}
$$

The azimuthal magnetic field is localized in the mantle inside the quadrupole current loop and does not exert an influence on the performance of the planetary electric generator. As follows from the expression (25), this field can not exceed $1.66 \delta_{c} \mathrm{G}$ under the terrestrial conditions and can be neglected outside the planetary surface.

The above consideration permits us make a conclusion that both the convective and quadrupole currents do not produce a substantial addition to magnetic field of the planetary core and can not exert a considerable influence on the electrodynamics of the planetary electric generator under the terrestrial conditions. It means that our initial assumption on the structure of the magnetic field was quite correct.

Now, we would like to summarize some results obtained in the article. A model of the planetary electric generator serving as one of the possible sources of the atmospheric electricity has been suggested. Developing our previous investigations (Bespalov and Chugunov, 1996; Bespalov et al., 1996), we have considered a more complete model of the planetary generator taking into account a complex inner structure of the planet. It was assumed that there is a uniformly magnetized core inside the planet and also angular velocity of the core generally is not equal to angular velocity of the mantle. This difference 
of the angular velocities leads to change of a global voltage applied to the atmospheric layer in comparison with the case of co-rotation between the core and mantle: the global voltage increases, if angular velocity of the core exceeds the angular velocity of the mantle, and decreases in the opposite case.

The main goal of the present article was to investigate an influence of complex inner structure of the planet on parameters of the global current circuit. To simplify the calculations and highlight the most important effects, the case of uniform atmospheric conductivity was studied. Of course, considered above model of the planetary electric generator can not be directly and completely applied to the Earth's conditions. First, we did not take into account differential rotation of the planetary fluid core. Second, atmospheric conductivity was assumed to be homogeneous in the model, whereas conductivity of the terrestrial atmosphere substantially depends on altitude over the planetary surface (Volland, 1987). As shown in our previous article (Bespalov et al., 1996), altitude inhomogeneity of the terrestrial atmospheric conductivity exerts an important influence on the electric field and current density distributions in the atmosphere. Latitude dependence of the atmospheric conductivity, which can be connected with differences of physical conditions near the pole and at the equator of the planet and also change parameters of the global atmospheric electricity, was not also taken into account in the model. Finally, it should be pointed out that to obtain a more complete model of the terrestrial atmospheric electricity, one must also take into account both the effect of powerful equatorial upward currents connected with the thunderstorms and complex structure of the plasma envelope, where plasmasphere co-rotating with the Earth exists.

One can see that, in application to the terrestrial conditions, the above model of the planetary electric generator is simplified in many aspects. Nevertheless, this model gives a voltage between the Earth's surface and upper atmosphere about $U_{\text {eff }} \approx 95 \mathrm{kV}$, whereas experimentally observed voltage is about $3 \cdot 10^{5} \mathrm{~V}$ (Mühleisen, 1977). It is shown that a contribution of the core-mantle relative rotation into the global ground-ionosphere voltage can be neglected under the terresrtial conditions, but can be substential at Jupiter. One can make a conclusion that since the elaborated mechanism of atmospheric electricity contributes about one third of the observed voltage, the suggested mechanism of generation of the atmospheric electricity is substantial enough and must be considered equally with such well-known sources as thunderstorm activity and magnetospheric macro processes.

Acknowledgments. The work has been supported partly by Russian Foundation for Fundamental Researches grant No. 95-02-04274a, partly by INTAS grant No. 94-1054 and partly by a fellowship of Tomalla Foundation under the research program of International Center for Fundamental Physics in Moscow (S. Davydenko).

\section{References}

Bespalov, P.A. and Yu.V. Chugunov, Planetary plasma envelope rotation and the nature of atmospheric electricity, J. Atm. Terr. Phys., $\underline{58}, 601-604,1996$.

Bespalov, P.A., Yu.V. Chugunov and S.S. Davydenko, Planetary electric generator under fair-weather condition with altitude-dependent atmospheric conductivity, J. Atm. Terr. Phys., 58, 605-611, 1996.

Landau, L.D., E.M. Lifshitz and L.P. Pitaevskii, Electrodynamics of Continuous Media, Pergamon Press, Oxford, 1984.

Lefftz, M., R. Sabadini and H. Legros, Mantle rheology, viscomagnetic coupling at the core-mantle boundary and differential rotation of the core induced by pleistocenic deglaciation, Geophys. J. Int., 117, 1-18, 1994.

Love, J.J. and J. Bloxham, Electromagnetic coupling and the toroidal magnetic field at the core-mantle boundary, Geophys. J. Int., 117, 235-256, 1994. 
Mühleisen, R., The Global Circuit and Its Parameters, in Electrical Processes in Atmospheres, ed. by H. Dolezalek, R. Reiter and H.E. Landsberg, Steinkopff, Darmstadt, 467-476, 1977.

Panofsky, W.K.H. and M. Phillips, Classical Electricity and Magnetism, Addison-Wesley Publ. Co., Inc., Cambridge, 1969.

Parker, E.N., Cosmical Magnetic Fields. Their Origin and Their Activity, Clarendon Press, Oxford, 1979.

Reiter, R., Fields, Currents and Aerosols in the Lower Troposphere, Amerind Publishing Co. Pvt. Ltd., New Dehli, pp.714, 1985.

Stevenson, D.J. and E.E. Salpeter, Models of the Jovian Interior, in Jupiter. Studies of the Interior, Atmosphere, Magnetosphere and Satellites, ed. by T. Gehrels, the University of Árizona Press, Tucson, Arizona, 1976.

Volland, H., Global, Quasi-Static Electric Fields in the Earth's Environment, in Electrical Processes in Atmospheres, ed. by H. Dolezalek, R. Reiter and H.E. Landsberg, Steinkopff, Darmstadt, 509-528, 1977.

Volland, H., Electromagnetic coupling between lower and upper atmosphere, Physica Scripta, T18, 289-297, 1987.

Wilson, C.T.R., The electric field of a thunderstorm cloud and some of its effects, Proc. Phil. Soc., London, 37, 320, 1925.

(Received December 19, 1996; revised March 29, 1996; accepted April 5, 1996.) 